\title{
Distribuição de espécies de borrachudos (Diptera: Simuliidae) do Espírito Santo, Brasil
}

\author{
Karina Bertazo $^{1,2,4}$, Claudiney Biral dos Santos ${ }^{I}$, Israel de Souza Pinto ${ }^{I}$, \\ Adelson Luiz Ferreira ${ }^{1}$, Aloísio Falqueto ${ }^{1}$ \& Mateus Pepinelli ${ }^{3}$ \\ ${ }^{1}$ Unidade de Medicina Tropical, Centro de Ciências da Saúde, \\ Universidade Federal do Espírito Santo - UFES, Av. Marechal Campos, 1.468, \\ CEP 29043-900, Vitória, ES, Brasil \\ ${ }^{2}$ Programa de Pós-graduação em Biodiversidade Tropical, Centro Universitário Norte do Espírito Santo, \\ Universidade Federal do Espírito Santo - UFES, Rod. BR101 Norte, Km 60, \\ CEP 20932-540, São Mateus, ES, Brasil \\ ${ }^{3}$ Laboratório de Entomologia Aquática, Faculdade de Filosofia, Ciências e Letras de Ribeirão Preto, \\ Universidade de São Paulo - USP, Ribeirão Preto, SP, Brasil \\ ${ }^{4}$ Autor para correspondência: Karina Bertazo, e-mail: karina_bertazo@hotmail.com
}

BERTAZO, K., SANTOS, C.B., PINTO, I.S., FERREIRA, A.L., FALQUETO, A. \& PEPINELLI, M. Distribution of black flies (Diptera: Simuliidae) in the State of Espírito Santo, Brazil. Biota Neotrop. 10(3): http:// www.biotaneotropica.org.br/v10n3/en/abstract?article+bn02110032010.

Abstract: We conducted a freshwater survey from 2004 to 2008 in several localities of Espírito Santo, Brazil. In total, 66 streams and rivers were sampled in the 12 hydrographic basins of the state. We collected and identified 17 species of which nine represent new records (Simulium pertinax, S. jujuyense, S. rubrithorax, S. subnigrum, S. travassosi, S. spinibranchium, S. hirtipupa, S. lutzianum e $S$. anamariae). These findings doubled the number of records of black fly species to the State of Espírito Santo, Brazil.

Keywords: black fly, macroinvertebrates, Atlantic Forest.

BERTAZO, K., SANTOS, C.B., PINTO, I.S., FERREIRA, A.L., FALQUETO, A. \& PEPINELLI, M. Distribuição de espécies de borrachudos (Diptera: Simuliidae) do Espírito Santo, Brasil. Biota Neotrop. 10(3): http:// www.biotaneotropica.org.br/v10n3/pt/abstract?article+bn02110032010.

Resumo: Entre os anos de 2004 e 2008 foram realizadas coletas de Simuliidae em várias localidades no Estado do Espírito Santo, Brasil. No total, 66 córregos e rios das doze principais bacias hidrográficas do estado foram amostrados. Dezessete espécies foram coletadas, sendo que nove delas representam ocorrências novas para o estado (Simulium pertinax, S. jujuyense, S. rubrithorax, S. subnigrum, S. travassosi, S. spinibranchium, S. hirtipupa, S. lutzianum e S. anamariae), duplicando o número de espécies conhecidas no estado.

Palavras-chave: simulídeos, macroinvertebrados, Mata Atlântica. 


\section{Introdução}

Os simulídeos (Diptera, Simuliidae), popularmente denominados "borrachudos", são insetos cujas fêmeas possuem hábito hematófago. Algumas espécies são antropofílicas podendo causar alergias e transmitir doenças durante o repasto sanguíneo (Strieder \& Corseuil 1992).

Nas Américas, os simulídeos são incriminados como os principais vetores da onconcercose, que tem como agente etiológico a Onchocerca volvulus (Leuckart) (Romero-Sá \& Maia-Herzog 2003). No Brasil, casos da doença foram registrados nos Estados de Roraima e Amazonas, sempre associados à presença desses dípteros (Cerqueira 1959, Moraes \& Chaves 1974, Shelley \& Coscarón 2001). As espécies Simulium incrustatum Lutz, S. guianense Wise, S. oyapockense Floch $\&$ Abonnenc e $S$. roraimense Nunes de Melo são apontadas como vetores (ou suspeitas de participarem da transmissão) da $O$. volvulus (Shelley 1988, Maia-Herzog et al. 1999).

Nas Américas são conhecidas cerca de 550 espécies de simulídeos, dentre as quais 92 ocorrem no Brasil (Adler \& Crosskey 2010, Hamada et al. 2010). O Estado de São Paulo apresenta o maior número de registros, com 52 espécies. No Estado do Espírito Santo são escassos os trabalhos sobre a fauna de simulídeos, com apenas oito espécies registradas, de modo geral, com base em resultados de coletas eventuais (Coscarón 1991). O conhecimento sobre a distribuição geográfica dos simulídeos é fundamental para o planejamento das ações de controle sobre espécies causadoras de incômodos à população ou transmissoras de patógenos (Amaral-Calvão \& Maia-Herzog 2003).

Diante da necessidade de conhecer a biodiversidade e da escassez de informações sobre simulídeos no Estado do Espírito Santo, foi realizado o presente estudo, abrangendo as principais bacias hidrográficas do estado, com intuito de atualizar o conhecimento sobre a riqueza da fauna de Simuliidae e a sua distribuição geográfica.

\section{Material e Métodos}

\section{1. Área de estudo}

O Estado do Espírito Santo possui pouco mais de $46.000 \mathrm{~km}^{2} \mathrm{de}$ extensão e é constituído de 78 municípios e 12 bacias hidrográficas (Figura 1). O clima predominante é o tropical de altitude com temperatura média superior a $22{ }^{\circ} \mathrm{C}$. Representa um dos menores estados brasileiros e abrange duas regiões distintas: planície, em sua maioria, localizada na região litorânea, representando $40 \%$ da área total do estado, e planalto com até $2.890 \mathrm{~m}$ de altitude, como o Pico da Bandeira, em geral situados mais para o interior do continente.

O Estado do Espírito Santo faz parte de uma das áreas mais importantes para a conservação da biodiversidade do planeta, o Corredor Central da Mata Atlântica, que abrange também o Sul do Estado da Bahia e algumas áreas do Leste do Estado de Minas Gerais, representando um dos maiores centros de endemismo da Mata Atlântica (Feitoza 2001).

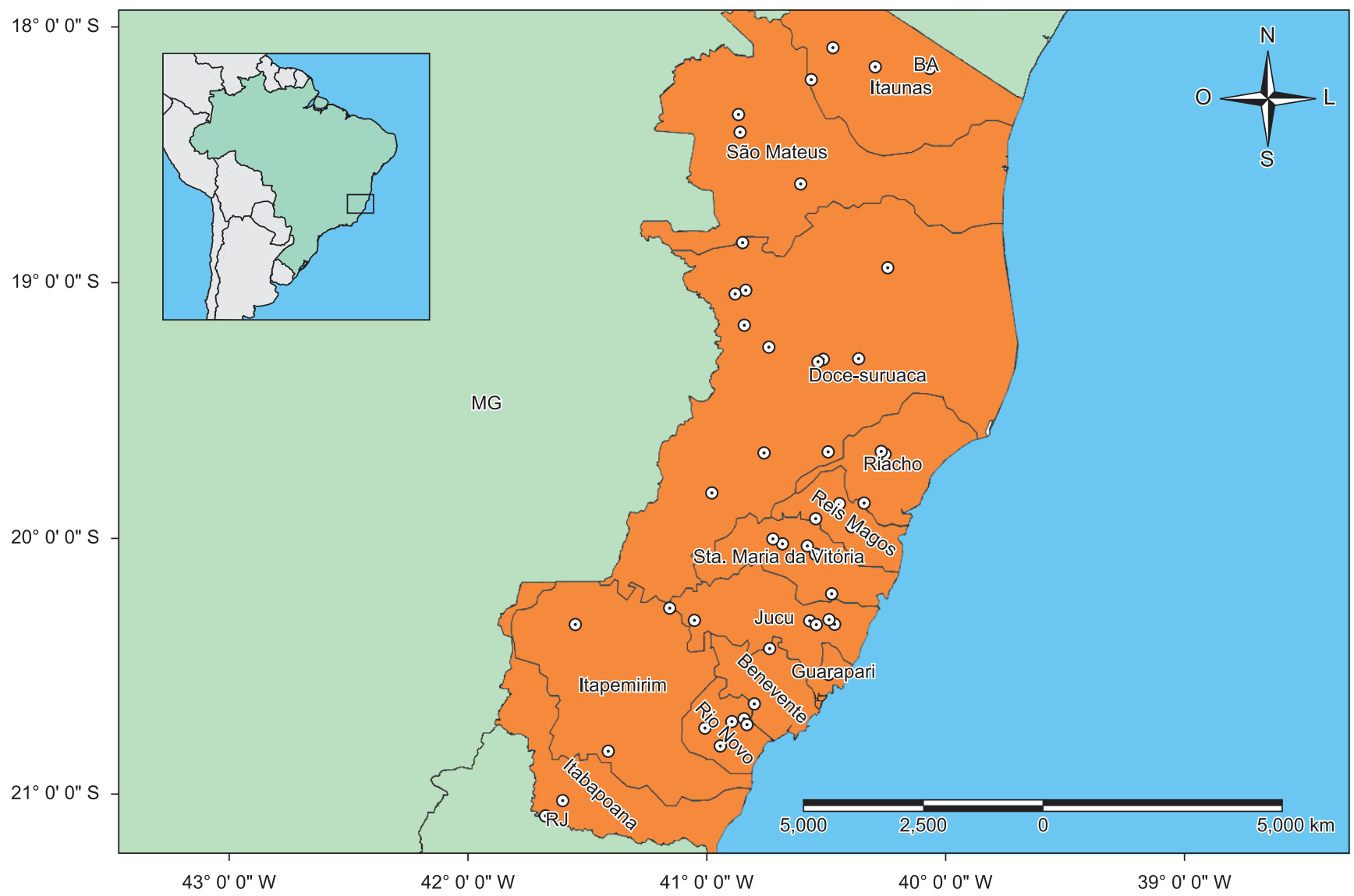

Figura 1. Mapa do Estado do Espírito Santo, Brasil, e suas principais bacias hidrográficas.

Figure 1. Map of the State of Espítito Santo, Brazil showing its hydrographic basins. 


\section{Coleta de Simuliidae}

Entre os anos de 2004 e 2008 foram realizadas 66 coletas de adultos e imaturos de Simuliidae em 30 municípios distribuídos nas 12 bacias hidrográficas do estado. Os espécimes adultos foram capturados durante sua atividade de hematofagia com capturador manual de sucção do tipo Castro e conservados em álcool 70\%. As pupas foram coletadas com o auxílio de pinça, diretamente de folhas, pedras e outros substratos e fixadas em álcool $70 \%$. Adultos farados foram mantidos em frascos isolados até a emergência dos adultos. Alguns adultos foram diafanizados, dissecados e montados entre lâmina e lamínula, segundo técnica descrita por Barreto \& Coutinho (1940), a fim de ser observada sua morfologia interna. Outros foram montados em alfinete para o exame da morfologia externa. As exúvias das pupas também foram montadas entre lâmina e lamínula.

Para a identificação foi utilizada bibliografia especializada e trabalhos originais de descrições de espécies (e.g. Coscarón \& Wygodzinsky 1984, Coscarón 1987, 1991, Shelley et al. 1997, 2004). Os espécimes foram depositados na Coleção Entomológica da Unidade de Medicina Tropical da Universidade Federal do Espírito Santo, Brasil (CEUMT). Informações sobre a diversidade de espécies de Simuliidae nos estados brasileiros foram compiladas a partir do inventário taxonômico e geográfico mundial de Simuliidae (Adler \& Crosskey 2010).

\section{Resultados e Discussão}

Foram coletadas 17 espécies de simulídeos. Destas, nove representam novas ocorrências para o Estado do Espírito Santo (Simulium pertinax Kollar, 1832; S. jujuyense Paterson \& Shannon, 1927; S. rubrithorax Lutz, 1909; S. travassosi D’Andretta \& D'Andretta, 1947; S. subnigrum Lutz, 1910; S. spinibranchium
Lutz, 1910; S. anamariae Vulcano, 1962; S. hirtipupa Lutz, 1910 e S. lutzianum Pinto, 1932). As oito espécies já registradas para o estado e recoletadas nesse estudo são: Simulium nigrimanun Macquart, 1832; S. incrustatum Lutz, 1910; S. inaequale Paterson \& Shannon, 1927; S. subpallidum Lutz, 1910; S. perflavum Roubaud, 1906; S. brachycladum Lutz \& Pinto, 1932; S. guianense Wise, 1911 e S. limbatum Knab, 1905 (Adler \& Crosskey, 2010).

As espécies antropofílicas que importunam o ser humano de forma mais agressiva foram as mais amplamente distribuídas no Estado do Espírito Santo. Simulium pertinax foi coletada em todas as 12 bacias hidrográficas, seguida por $S$. nigrimanum e $S$. incrustatum, localizadas em 11 e oito bacias, respectivamente (Tabela 1). De acordo com o inventário mundial de Simuliidae (Adler \& Crosskey 2010) as espécies $S$. pertinax, S. nigrimanum e $S$. incrustatum estão registradas para 15, 11 e 19 Estados (incluindo o Distrito Federal) do Brasil, respectivamente, o que mostra a ampla distribuição dessas espécies antropofílicas. Em localidades onde os moradores relataram espontaneamente o grande importuno causado pela picada dos "borrachudos", foi constatada a presença das espécies $S$. pertinax e S. nigrimanum, com destaque para o município de Iconha, Rio Iconha, Salto Grande e o município de Alfredo Chaves no ribeirão do Cristo. Coscarón (1991) cita $S$. pertinax como a espécie que mais provoca incômodo por sua picada. No litoral Norte do Estado de São Paulo e na região Sul do Brasil $S$. pertinax é a principal espécie que exerce influência negativa na qualidade de vida das pessoas e causa prejuízos econômicos na pecuária e no turismo (Araújo-Coutinho et al. 1988; Strieder \& Corseuil 1992).

As espécies de simulídeos registradas no Estado do Espírito Santo são também encontradas nos estados vizinhos. Rio de Janeiro, Minas Gerais e Bahia possuem, respectivamente, 15, 14 e 12 espécies em comum com o Estado do Espírito Santo. Apenas uma espécie

Tabela 1. Distribuição por bacia hidrográfica das espécies de Simuliidae no Estado do Espírito Santo, Brasil. O número entre parênteses indica a quantidade de córregos amostrados em cada bacia hidrográfica.

Table 1. Distribution of black fly species per hydrographic basin in the State of Espírito Santo, Brazil. For each basin, the number of streams sampled is given in parenthesis.

\begin{tabular}{|c|c|c|c|c|c|c|c|c|c|c|c|c|}
\hline Espécie & $\begin{array}{l}\text { Itaúnas } \\
\text { (3) }\end{array}$ & $\begin{array}{c}\text { São } \\
\text { Mateus } \\
(6)\end{array}$ & $\begin{array}{c}\text { Doce- } \\
\text { Suruaca } \\
\text { (19) }\end{array}$ & $\begin{array}{c}\text { Riacho } \\
\text { (6) }\end{array}$ & $\begin{array}{c}\text { Reis } \\
\text { Magos } \\
\text { (4) }\end{array}$ & $\begin{array}{c}\text { Santa } \\
\text { Maria da } \\
\text { Vitória } \\
\text { (5) }\end{array}$ & $\begin{array}{c}\text { Jucu } \\
(7)\end{array}$ & $\begin{array}{c}\text { Guarapari } \\
\text { (2) }\end{array}$ & $\begin{array}{c}\text { Benevente } \\
\text { (3) }\end{array}$ & $\begin{array}{c}\text { Rio } \\
\text { Novo } \\
(6)\end{array}$ & $\begin{array}{l}\text { Itapemirim } \\
\text { (3) }\end{array}$ & $\begin{array}{c}\text { Itabapoana } \\
\text { (2) }\end{array}$ \\
\hline *S. pertinax & $\mathrm{X}$ & $\mathrm{X}$ & $\mathrm{X}$ & $\mathrm{X}$ & $\mathrm{X}$ & $\mathrm{X}$ & $\mathrm{X}$ & $\mathrm{X}$ & $\mathrm{X}$ & $\mathrm{X}$ & $\mathrm{X}$ & $\mathrm{X}$ \\
\hline S. nigrimanum & $\mathrm{X}$ & - & $\mathrm{X}$ & $X$ & $\mathrm{X}$ & $X$ & $\mathrm{X}$ & $X$ & $\mathrm{X}$ & $\mathrm{X}$ & $X$ & $X$ \\
\hline S. incrustatum & $\mathrm{X}$ & $\mathrm{X}$ & $\mathrm{X}$ & $\mathrm{X}$ & - & $\mathrm{X}$ & $\mathrm{X}$ & - & $\mathrm{X}$ & $\mathrm{X}$ & - & - \\
\hline S. inaequale & - & - & - & $\mathrm{X}$ & - & $\mathrm{X}$ & - & - & $\mathrm{X}$ & $X$ & $\mathrm{X}$ & - \\
\hline S. subpallidum & - & - & - & $\mathrm{X}$ & $\mathrm{X}$ & - & - & - & - & - & $\mathrm{X}$ & - \\
\hline S. perflavum & - & - & - & $\mathrm{X}$ & - & - & - & - & - & - & $\mathrm{X}$ & - \\
\hline S. brachycladum & - & - & - & $\mathrm{X}$ & - & - & - & - & - & - & - & - \\
\hline *S. jujuyense & $\mathrm{X}$ & $X$ & - & - & - & - & - & - & $\mathrm{X}$ & - & - & - \\
\hline *S. rubrithorax & - & - & - & - & $\mathrm{X}$ & - & - & - & - & - & - & - \\
\hline *S. travassosi & - & - & - & - & - & - & $\mathrm{X}$ & - & - & - & - & - \\
\hline S. guianense & - & - & $X$ & - & - & - & - & - & - & - & - & - \\
\hline *S. subnigrum & - & - & - & - & - & - & - & - & $\mathrm{X}$ & - & - & - \\
\hline *S. spinibranchium & - & - & - & $\mathrm{X}$ & - & - & - & - & - & - & - & - \\
\hline *S. lutzianum & - & - & - & - & - & - & - & - & $X$ & $\mathrm{X}$ & - & - \\
\hline *S. anamariae & - & - & - & - & - & - & - & - & - & $\mathrm{X}$ & - & - \\
\hline S. limbatum & - & - & - & $\mathrm{X}$ & - & - & - & - & - & - & - & - \\
\hline *S. hirtipupa & - & - & $\mathrm{X}$ & - & - & - & - & - & - & - & - & - \\
\hline
\end{tabular}

*Ocorrências novas de simulídeos no estado. 
coletada, S. lutzianum, ainda não foi encontrada nos estados vizinhos. De acordo com o inventário taxonômico e geográfico mundial de Simuliidae (Adler \& Crosskey 2010), S. lutzianum está distribuída nos Estados do Sul do Brasil, Goiás e Rondônia. No entanto, Pepinelli et al. (2005) encontraram-na também no Estado de São Paulo, no Parque Estadual de Intervales.

O fato do presente estudo ter duplicado o número de espécies registradas pode ser explicado pela escassez de estudos realizados no Estado do Espírito Santo. De acordo com inventário de Adler \& Crosskey (2010), no Brasil a Região Sudeste possui o maior número de espécies registradas (57), seguida pelas Regiões Norte (47), Sul (38), Centro-Oeste (30) e Nordeste (23). Mesmo passando a contar com registro de 17 espécies, o Estado do Espírito Santo ainda possui um número muito menor de registros de espécies de Simuliidae em comparação com os Estados de Minas Gerais, Rio de Janeiro e São Paulo, cada um contando com 36, 37 e 52 espécies respectivamente.

O presente estudo amplia o conhecimento sobre a distribuição geográfica de simulídeos no Brasil, registrando para o Estado do Espírito Santo, pela primeira vez, a ocorrência de nove espécies. Trabalhos futuros poderão acrescentar mais espécies e ampliar a área geográfica de ocorrências das já registradas.

\section{Agradecimentos}

Ao Gustavo Rocha Leite pela contribuição na elaboração do mapa. Ao Senhor Ricardo Del Carro e a Senhora Maria Odete Bertazo fundamentais na ajuda nos trabalhos de campo.

\section{Referências Bibliográficas}

ADLER, P. \& CROSSKEY, R.W. 2010. World Black Flies (Diptera: Simuliidae): a comprehensive revision of the taxonomic and geographical inventory. 112p. http://entweb.clemson.edu/biomia/pdfs/blackflyinventory.pdf

AMARAL-CALVÃO, A.M.R. \& MAIA-HERZOG, M. 2003. Coleção de Simulideos (Diptera, Simuliidae) de Adolpho Lutz, sua História e Importância. Hist. Cienc. Saude 10(1):259-271.

ARAÚJO-COUTINHO, A., MAIA-HERZOG, M. \& SOUZA, B.C. 1988. Levantamento das espécies do gênero Simulium Latreille (Diptera, Simuliidae) no litoral norte do Estado de São Paulo. Rev. Bras. Entomol. 32(1):11-17.

BARRETO, M.P. \& COUTINHO, J.O. 1940. Processos de captura, dissecação e montagem de flebótomos. Annual Faculdade Medicina. São Paulo, 16:173-187

CERQUEIRA, N.L. 1959. Sôbre a transmissão da Mansonella Ozzardi. J. Bras. Med. 5(29).
COSCARÓN, S. \& WYGODZINSKY, P. 1984. Notas sobre simúlidos neotropicales Vll. Sobre los subgêneros Psaroniocompsa Enderlein y Inaequalium, subgen. nov. Arq. Zool. 31(2):35-103.

COSCARÓN, S. 1987. El Gênero Simulium Latreille em la Región Neotropical: Análisis de los Grupos Supraespecificos, espécies que los Integran y Distribuición Geográfica (Simuliidae, Diptera). Museu Paranaense Emílio Goeldi, 112p.

COSCARÓN, S. 1991. Fauna de água dulce de la Republica Argentina. FECIC. Bs. Aires. Insecta, Diptera, Simuliidae 38(2):295.

FEITOZA, L.R., STOCKING, M. \& RESENDE, M. 2001. Natural resources information systems for rural development: approaches for Espírito Santo State, Brazil. Incaper, Vitória, 223p.

HAMADA, N., PEPINELLI, M., MATTOS-GLÓRIA, A.A. \& LUZ, S.L.B. 2010. A new black fly species from Brazil, closely related to Simulium guianense Wise (Diptera, Simuliidae), revealed by morphology and DNA barcoding. Zootaxa 2428:22-36.

MAIA-HERZOG, M., SHELLEY, A.J., BRADLEY, J.E., LUNA DIAS, A.P.A., CALVÃO, R.H.S., LOWRY, C., CAMARGO, M., POST, R.J. \& COELHO, G.E. 1999. Discovery of a new focus of human onchocerciasis in Central Brazil. T. Roy. Soc. Trop. Med. H. 93:235-239.

MORAES, M.A.P. \& CHAVES, G.M. 1974. Oncocercose no Brasil. Novos achados entre os índios Ianomâmis. Bol. Of. Sanit. Panam. 1-5.

PEPINELLI, M., TRIVINHO-STRIXINO, S. \& HAMADA, N. 2005. Imaturos de Simuliidae (Diptera, Nematocera) e caracterização de seus criadouros no Parque Estadual Intervales, São Paulo, Brasil. Rev. Bras. Entomol. 49(4):527-530.

ROMERO-SÁ, M. \& MAIA-HERZOG, M. 2003. Doença de além-mar: estudos comparativos da oncocercose na América Latina e África. Hist. Cienc. Saude 10(01):251-258.

SHELLEY, A.J. \& COSCARÓN, S. 2001. Simuliid blackflies (Diptera: Simuliidae) and Ceratopogonid midges (Diptera: Ceratopogonidae) as vectors of Mansonella ozzardi (Nematoda: Onchocercidae) in northern Argentina. Mem. Inst. Oswaldo Cruz 96(4):451-458.

SHELLEY, A.J. 1988. Vector Aspects of the Epidemiology of Onchocercosis in Latin American. Annu Rev Entomol. 33:337-366.

SHELLEY, A.J., HERNANDÉZ, L.M. \& DAVIES, J.B. 2004. Blackflies (Diptera: Simuliidae) of southern Guyana with keys for the identification of adults and pupae - a review. Mem. Inst. Oswaldo Cruz 99(5):443-470.

SHELLEY, A.J., LOWRY, C.A., MAIA-HERZOG, M., LUNA DIAS, A.P.A. \& MORAES, M.AP. 1997. Biosystematic studies on the Simullidae of the Amazonia onchocerciasis focus. Bull. Br. Mus. Nat. Hist. 66:1-120.

STRIEDER, M.N. \& CORSEULI, E. 1992. Atividades de hematofagia em Simuliidae (Diptera, Nematocera) na Picada Verão, Sapiranga, RS-Brasil. Acta Biol. Leopoldensia 14(2):75-98.

Recebido em 02/03/2010

Versão reformulada recebida em 07/07/2010

Publicado em 27/07/2010 